\title{
Manifestations of a low-frequency sound of unknown origin perceived worldwide, also known as "the Hum" or the "Taos Hum"
}

Franz Günter Frosch ${ }^{1}$

\begin{abstract}
Introduction: "The Hum" and "Taos Hum" are the common terms used for an annoying low-frequency sound of unknown and seemingly mysterious external causes. The Hum is a phenomenon occurring worldwide that affects approximately $2 \%$ of the population, called hearers or hummers. Objective: This study investigates the different manifestations of the Hum based on questionnaires tailor-made for hearers, with the aim to demystify this phenomenon. Material and Methods: Questionnaires completed by 162 hearers were evaluated, with a focus on three often-reported features: whether their Hum forms beats with external sounds, whether head rotations remove their Hum, and if air travel produces a time lag until the Hum reappears. Results and Conclusions: The presence of at least one of the three features is typical of the Hum for $73 \%$ hearers. The three features are statistically significant dependent on each other. Hum-oscillations are typically influenced by head rotation and by sounds and are most likely located in the semicircular canals and the cochlea. Therefore, for the majority of hearers, the Hum may represent a rare form of tinnitus that has nothing to do with external sounds. The remaining group doesn't experience any feature of a typical hearer.
\end{abstract}

Keywords: noise, otoacoustic emissions spontaneous, quality of life, tinnitus, questionnaires.

\footnotetext{
${ }^{1}$ Private Initiative Brummton, Bad Dürkheim, Germany. E-mail: frosch.com@t-online.de Institution: Dr. Franz Frosch, Private Initiative Brummton, Bad Dürkheim, Germany Send correspondence to:

Franz Günter Frosch

Auf dem Köppel 1 Nr.11, D-67098 Bad Dürkheim, Germany

Paper submitted to the RBCMS-SGP (Publishing Management System) on April 30, 2016;

and accepted on July 5, 2016. cod. 238
} 


\section{INTRODUCTION}

The perception of an annoying low-frequency sound of unknown origin, also known as "the Hum" or the "Taos Hum", is found to be a worldwide phenomenon that affects approximately $2 \%$ of the population, called hearers or hummers. Although it is often believed that hearers hear the same mysterious sound, frequency matches in Taos demonstrate that the Hum does not consist of one Hum frequency identical to each hearer, but rather of different frequencies ranging from $30 \mathrm{~Hz}$ to $80 \mathrm{~Hz}$ that are different, even for hearers in direct proximity ${ }^{1}$.

Hearers report that their Hum may form beats with, lock into, and match the frequency of an external sound (ES); that they experience a time lag of two to three days until their Hum reappears after longer air travel; and that they can stop their Hum during purposeful head movements. Such observations are not commonly reported for tinnitus, which is the main reason why these force-interactive hums (FIHs) very often are not assigned to tinnitus but rather to other somehow mysterious external sources ${ }^{2}$.

The term acoustic beat is classically used to describe the interference between two ESs of slightly different frequencies, perceived as periodic variations of their volumes at a rate corresponding to the difference of the two frequencies. This kind of interference has long been familiar because beats can be generated in almost all human ears. The Hum can also beat with an already present ES as if it were an ES itself.

Interference between two forces only occurs as long as the same process and the same system are involved simultaneously. If the forces involve two separate receptor elements, a pattern of interference cannot arise. Further, it is a physical impossibility for two entirely different forms of energy to interact in this manner; that is, it is inconceivable that beats could be produced by interference of electrical and mechanical forces. This principle is called the superposition principle or superposition property, and the terminology suggests the Hum to be a second ES, which beats with the present ES.

The aim of this study is to discuss the reasons for this unusual behavior of the Hum on the basis of replies to questionnaires customized for hearers, and to localize the FIH oscillations.

\section{MATERIALS AND METHODS}

The study is based on 162 questionnaires. They differ from the usual questionnaires used for tinnitus because they contain questions with a special attention on frequently reported observations of hearers. The questionnaires have been designed under the leadership of a group of scientists who have been personally involved in Hum phenomenon for several years. The questionnaires could be downloaded from a homepage or were sent via email or letter. All questionnaires that arrived within a given period of time are included in the study.

The evaluations were carried out in 2015 , and the focus was on the three frequently reported features: whether their Hum forms beats with ESs, whether head rotations remove their Hum, and whether air travel produces a time lag until the Hum reappears.

The Van der Pol-oscillator is the simplest model used to simulate nonlinear biological oscillations and a tool to proof the nonlinearity of SIHs. It closely imitates biological phenomena and has been used successfully to simulate spontaneous otoacoustic emissions ${ }^{3}$. The typical interactions of the forced Van der Pol oscillator, the periodic pulling with, and synchronization into external sounds are used to prove the internal origin of $\mathrm{SIHs}^{4}$.

Beats between an ES and the Hum are used as a tool to determine the locations of the Hum-oscillations because beats between one ES with one internal soundoscillator may behave differently to beats between two ESs. They may generate beat-frequencies that are pulling towards the ES to an extent that depends on the frequency difference, while beats between two ESs interact linearly. This phenomenon is well known for oscillators in the ear that can be simulated as forced Van der Pol-oscillators ${ }^{3}$.

\section{RESULTS}

\section{Basic information about hearers}

In the study, 83 males and 79 females with an age range from 9 to 84 years (average \pm SD of $48 \pm 11$ ) completed the questionnaire survey. At the time of the data collection participants had been experiencing their Hum for an average of 6 years. For the majority of hearers, the Hum does not change its pitch, and it is heard only in silent surroundings.

Sixty-five percent of hearers describe their Hum as the sound of a truck idling in the distance, $27 \%$ as the humming sound of the transformer working, $14 \%$ as the droning of a propeller plane, and $11 \%$ the chugging of a fishing boat. Multiple responses were possible. The location of the Hum is perceived by $26 \%$ in both ears, by $26 \%$ it alternates between the ears, by $15 \%$ it is heard in the head, by $17 \%$ in the left ear, and by $9 \%$ in the right ear.

Otologists certified a healthy ear for $77 \%$ of hearers, and $86 \%$ evaluated their hearing ability as normal to above average. Further, 15 participants matched their Hum with the sound of a generator plus headphones as shown in Table 1, which confirms the findings of Mullins and Kelly ${ }^{1}$ that the Hum cannot be a suspected external sound, audible only to sensitive hearers. In this case 
hearers in the immediate vicinity would have matched the generators to the same frequency, and no difference would have been matched between the right and left ear of each individual hearer.

Table 1. Measures and locations of Hum impressions.

\begin{tabular}{lccc}
\hline Person & Left Ear $(\mathrm{Hz})$ & Right Ear $(\mathrm{Hz})$ & Impression \\
\hline DB & 32 & - & left ear \\
DS & 70 & - & left ear \\
EC & 65 & - & left ear \\
FF & $56-58$ & $65-68$ & head \\
FV & 75 & 78 & head \\
FS & - & 30 & right ear \\
HA & 37 & 38 & head \\
HF & $65-68$ & $65-69$ & both ears \\
KL & 65 & 57 & both ears \\
KB & - & 40 & right ear \\
LG & $35-45$ & $35-45$ & both ears \\
MA & 37 & 37 & head \\
RA & 70 & 70 & both ears \\
ST & - & 50 & right ear \\
SM & $75-80$ & $75-80$ & both ears \\
\hline
\end{tabular}

Additionally, it demonstrates an interesting relationship: when the Hum is present only in one ear, the Hum impression is in the same ear. When the Hum can be measured in both ears, the impression is either in both ears or in the head. When sounds are presented to a listener dichotically, or one through each ear, binaural beats may occur. Depending on their frequency-differences, binaural beats may have negative effects on mood 5 .

Simultaneous Hum-oscillations in both ears may cause binaural beat interactions perceptible as volume fluctuations, vibrations, and the droning of the Hum. Binaural Hum-interactions may be the reason for the often reported body vibrations and the negative mood of many hearers.

\section{Main features of the Hum}

In $60 \%$ of hearers, the Hum interacts with sounds, and a quarter of them additionally report in detail that their Hum may form beats with, lock into, and match the frequency of an ES. A time lag of two to three days until the Hum reappears after longer air travel has been reported by $55 \%$ of hearers. Thirty-seven percent report a Hum that can stop during purposeful head movement; for half of them, this takes place exclusively by horizontal head rotation. The three main features of the Hum, being a sound-interacting Hum (SIH), a time-lag Hum (TLH), and a head-rotation stopped $\mathrm{Hum}(\mathrm{HRH})$ are statistically evaluated.
The chi-squared $\left(\mathrm{Chi}^{2}\right)$ test of independence shows a strong stochastic dependence at $p<0.01$ for the simultaneous occurrence of SIHs and TLHs $\left(\mathrm{Chi}^{2}=9.29\right)$ and for the simultaneous occurrence of $\mathrm{SIHs}$ and $\mathrm{HRHs}$ $\left(\mathrm{Chi}^{2}=8.42\right)$. The simultaneous occurrence of a TLH and an $\mathrm{HRH}$ appears coincidentally $\left(\mathrm{Chi}^{2}=1.01\right)$. These different mutual dependencies indicate a key function for SIHs.

The simultaneous presence of an $\mathrm{SIH}, \mathrm{a} \mathrm{TLH}$, and an $\mathrm{HRH}$ in the Hum of a single hearer would offer an ideal situation to test the dependencies and correlations of these three main features of the Hum; this rare simultaneous occurrence is only represented in $7 \%$ of hearers, as in the case report of Frosch ${ }^{4}$.

Cumulatively, we found an SIH or TLH for $66 \%$ and an $\mathrm{SIH}, \mathrm{TLH}$, or $\mathrm{HRH}$ for $73 \%$ of the hearers. Additional abnormalities of the Hum have not been observed by the hearers. This indicates that these three parameters of FIHs may completely describe the Hum. The remaining $27 \%$ do not fall under the definition of an $\mathrm{FIH}$ and should not be called hearers because their complaints are caused by completely different reasons that are not correlated with FIHs and do not fit into the typical reports on the Hum as a worldwide phenomenon.

\section{DISCUSSION}

Careful examinations of the manifold individual statements received from hearers in questionnaires, personal measurements on their own Hum, and contacts to self-help groups lead to the conviction that the oftenreported strange observations that sound, rotational forces, and longer air travel are influencing FIHs cannot be artifacts but must have something to do with the location and generation of FIHs.

Although the results of the questionnaires were obtained from a limited number of Hum sufferers (162), mainly from one geographical area, this number demonstrates a significant trend. Identical findings of individual hearers all over the world confirm this evaluation; and quantitative evaluations concerning these main features from third parties do not exist. Let us concentrate on the $73 \%$ of "real" hearers experiencing FIHs and assign their origins from the observations made on SIHs, TLHs and HRHs:

SIHs can be simulated as Van der Pol oscillators and their oscillations are supposed to be located in the cochlea. This kind of perceived sound is actively involved in the hearing process. TLHs cannot be used to define a location of the Hum as long as the receptors for the Hum-oscillations within the ear are not clearly localized. Undoubtedly, some unusual additional external influences may cause this strange effect. Possible causes are exposure to abrupt changes of atmospheric pressure or gravity, or to prolonged vibration and noise, all of 
which are known to affect the vestibular system. HRHs, however, can definitely be assigned to the receptors for detecting rotational forces, which are the vestibular hair cells in the cristae ampullaris of the semicircular canals.

When integrating these three kinds of FIHs, it can be concluded that there are two locations simultaneously involved in the generation of the Hum: the cochlea and the semicircular canals. These results are supported by the highly significant stochastic dependence of the simultaneous occurrence of SIHs and TLHs and of SIHs and $\mathrm{HRH}$. The $73 \%$ of $\mathrm{FIHs}$ cannot be caused by an ES, because no ES can force another ES into Van der Pol-interactions, no ES shows a delay in its audibility with/after the change of residence, and the Hum can be eliminated by head movements but the ES cannot.

Therefore, the Hum cannot be caused by any external source, and is not the result of any oscillation in the human body, being outside the direct signal path of the ear, such as the middle-ear myoclonus or other muscle contractions or reflexes of the human body that are called the causes of the Hum. Let us evaluate these findings:

There are many convincing arguments that the function of the cochlea is limited to sounds and that of the semicircular canals to head rotations. Neither tinnitus nor otoacoustic emissions are well-understood phenomena; they are mainly defined according to their clinical aspects, not on causes or mechanisms of production ${ }^{6}$. Audible spontaneous otoacoustic emissions and beats between an ES and tinnitus do not really fit into the definitions.

According to Vernon ${ }^{7}$, only $4 \%$ of subjects with tonal tinnitus can produce beats. Penner ${ }^{8}$ found that approximately $5 \%$ of all spontaneous otoacoustic emissions are also audible to the subject; however, the portion thereof that can produce beats has not been investigated.

An overlapping function has been reported for intense sounds that activate the utricular and saccular maculae neurons of the vestibule in addition to cochlear neurons ${ }^{9}$ but not for head rotations that influence acoustic neurons. However, the potential for acoustic stimuli to act on the balance sensors of the vestibular system and vice versa is greater than commonly appreciated because the auditory and vestibular sensory epithelia are housed in a common bony capsule, the labyrinth.

Acoustic sensation without endolymph flow in the semicircular canals may occur by longitudinal sound pressure waves acting on the vestibular hair cells of both sides of the three cristae identically and eliminating the influence of head movements ${ }^{10}$. Vestibular II hair cells are candidates to play an active part in the $\mathrm{FIH}$-oscillation for several reasons: They are sensitive to changes in hydrostatic pressure ${ }^{11}$, which may explain the observed time lag before the Hum reappears after longer air travel and may be the reason why it is temporarily not audible at other places. Air travel is known to be accompanied by strong and fast air-pressure changes that may have a prolonged effect on vestibular II hair cells.

Vestibular II hair cells respond to velocity but not to acceleration of head rotations ${ }^{12}$, which has been confirmed in a case report ${ }^{4}$ in which the Hum could be eliminated at an angular velocity above $306 \%$ s. Vestibular II hair cells interact with acoustic forces ${ }^{13}$; the found hearing sensitivity of single vestibular fibers of not better than $70 \mathrm{~dB}$ SPL may strongly be enhanced by averaging the signals of several hundred identical parallel vestibular neurons. When a signal is periodic and the noise is random, as a principle, the noise goes down as the square root of the number of averaged neurons.

Approximately 1300 myelinated and unmyelinated axons make contact through vestibulocochlear anastomosis ${ }^{14}$, a number sufficient to lower the vestibular hearing level by averaging below single digits. Acoustically responsive vestibular neurons with synapses on cochlear fibers may be an explanation for this ${ }^{15}$, as it seems that the hearing of the Hum involves the semicircular canals and the cochlea.

An external force can influence a Hum-oscillation only if it has direct access into the process of the oscillation. A Hum-oscillation that is influenced by head rotation and by sounds is most likely located in the semicircular canals and the cochlea, which interact with each other. It seems as if energy is generated in the semicircular canal and flows into the cochlea to support a self-sustained oscillation, and both get stopped during head rotation. The so-called Hum-interacting sites use information from both locations. Further studies are necessary to assign the functions and locations of the Hum-interacting sites and to identify the flow between both locations.

\section{CONCLUSIONS}

Based on the majority of replies to the questionnaires, the Hum is found to be sound interactive, sensitive to head rotations, and/or having a time lag of two to three days until it reappears after hearers' longer air travel. The presence of at least one of these three features can be taken as typical for the Hum.

Self-sustained oscillations similar to those observed with cochlear tinnitus or audible otoacoustic emissions may act in one or both ears and cause the Hum. For the majority of hearers the Hum may represent a rare form of tinnitus that has nothing to do with ESs. A small separate group is not sensitive to any of these features. Their complaints are caused by completely different reasons that are not correlated with any observations originally found with hearers. 


\section{REFERENCES}

1. Mullins JH, Kelly JP. The mystery of the Taos hum. Echoes. 1995;5(3):1,4,6.

2. Deming D. The hum: an anomalous sound heard around the world. J Sci Explor. 2004;18(4):571-95.

3. Talmadge CL, Long GR, Murphy WJ, Tubis A. Quantitative Evaluation of Limit-Cycle Oscillator Models of Spontaneous Otoacoustic Emissions. In: Dallos P, Geisler CD, Matthews JW, Ruggero MA, Steele C, eds. The Mechanics and Biophysics of Hearing. Proceedings of a conference held at the University of Wisconsin, Madison, WI, June 25-29, 1990. Springer Link, Lecture Notes in Biomathematics; 1990 p. 235-42.

4. Frosch FG. Hum and otoacoustic emissions may arise out of the same mechanisms. J Sci Explor. 2013;27(4):603-24.

5. Lane JD, Kasian SJ, Owens JE, Marsh GR. Binaural auditory beats affect vigilance performance and mood. Physiol Behav.1998;63(2):249-52.

6. Shulman A. Clinical Types of Tinnitus. In: Shulman A, ed. Tinnitus diagnosis/treatment. Philadelphia: Lea \& Febiger; 1991. p. 323-41.

7. Vernon JA. Common Errors in the Use of Masking for Relief of Tinnitus. In: Shulman A, ed. Tinnitus diagnosis/treatment. Philadelphia: Lea \& Febiger; 1991. p. 50-66.
8. Penner MJ. An estimate of the prevalence of tinnitus caused by spontaneous otoacoustic emissions. Arch Otolaryngol Head Neck Surg 1990;116(4):418-23.

9. Curthoys IS, Vulovic V. Vestibular primary afferent responses to sound and vibration in the guinea pig. Exp Brain Res. 2011;210(3-4):347-52.

10. Carey J, Amin N. Evolutionary changes in the cochlea and labyrinth: Solving the problem of sound transmission to the balance organs of the inner ear. Anat Rec A Discov Mol Cell Evol Biol. 2006;288(4):482-90.

11. Fraser PJ, Cruickshank SF, Shelmerdine RL. Hydrostatic pressure effects on vestibular hair cell afferents in fish and crustacea. J Vestib Res. 2003;13(4-6):235-42.

12. Kim KS, Minor LB, Santina CD, Lasker DM. Variation in response dynamics of regular and irregular vestibular-nerve afferents during sinusoidal head rotations and currents in the chinchilla. Exp Brain Res. 2011;210(3-4):643-9.

13. Dickman JD, Correia MJ. Responses of pigeon horizontal semicircular canal afferent fibers. II. High-frequency mechanical stimulation. J Neurophysiol. 1989;62(5):1102-12.

14. Arnesen AR. Fibre population of the vestibulocochlear anastomosis in humans. Acta Otolaryngol. 1984;98(5-6):501-18.

15. McCue MP, Guinan JJ Jr. Influence of efferent stimulation on acoustically responsive vestibular afferents in the cat. J Neurosci. 1994;14(10):6071-83. 